\title{
CLINICAL AND BIOCHEMICAL ANALYSIS FOR THE ADJUNCTIVE EFFECTS OF SIMVASTATIN AND METFORMIN IN THE TREATMENT OF CHRONIC PERIODONTITIS PATIENTS
}

\author{
Asem M. Kamel ${ }^{*}$, Hany G. Gobran ${ }^{* *}$, Ahmed O. Abdelrahman ${ }^{* * *}$ and Ahmed M. Hussein ${ }^{* * * *}$
}

\begin{abstract}
Background: adjunctive local periodontal therapy is one of the best options to improve the health of the pocket tissue. The comparison of local effects of simvastatin and metformin as aid in enhance the healing of periodontal tissue is the point of importance.
\end{abstract}

Methods: atotal of 45 systemically healthy patients with mild to moderate chronic periodontitis; which having a generalized pocket depth varied from $3 \mathrm{~mm}$ to $5 \mathrm{~mm}$. All patients handled by scaling and root planning and divided haphazardly into: group $\mathrm{I}$, as a control group $(\mathrm{n}=15)$, in which the patients treated by application of placebo gel; group II, $(n=15)$ treated with application of $1.2 \%$ simvastatin gel; group III, $(n=15)$, treated with application of $1 \%$ metformin gel. Clinical and biochemical criteria were collected at threshold 3, 6 and 9 months.

Results: an advancement in both plaque and gingival indexes was noticed in total 3 groups at 6 and 9 months. Plaque Index and Gingival Index showed decrement in groups II and III than group I. The cutback in probing depth and clinical attachment loss was higher in the group III than other groups. Significant minimization in both IL- 6 and TNF- $\alpha$ levels occurred from baseline to the end of measurement duration in all groups. Clear interaction was established amid IL- 6 and TNF- $\alpha$ heights.

Conclusion: metformin was comparatively better than simvastatin in respect of clinical and biochemical markers.

KEY WORDS: Simvastatin, Metformin, Periodontitis, Adjunctive therapy, IL-6, TNF- $\alpha$

* Department of Oral Medicine, Periodontology, Oral Diagnosis and Dental Radiology, Faculty of Dentistry, Al-Azhar University (Assiut Branch), Assiut, Egypt

** Department of Oral Biology, Faculty of Dentistry, Al-Azhar University (Boys Branch), Egypt

*** Department of Oral and Maxillofacial Surgery, Faculty of Dentistry, Al-Azhar University (Assiut Branch), Assiut, Egypt

**** Department of Oral and Maxillofacial Pathology, Faculty of Dentistry, Assiut University, Assiut, Egypt 


\section{INTRODUCTION}

The effect of all periodontal treatments depends upon elimination of the biofilm and decision of the periodontal pathogens which associated with the periodontal tissues to realize success ${ }^{(1)}$. Nonsurgical periodontal treatment target to accomplish a root surface that is biologically adequate for the reiteration of a healthy periodontal attachment ${ }^{(2)}$. Ancillary periodontal medicine may progress the performance of nonsurgical periodontal therapy, especially for high-risk patients ${ }^{(3)}$. Local distribution of the medication in the periodontal pocket is the favorite decision to remove the disease and get better the health of periodontal tissue. The dental pocket performance as a normal cistern and supplies simple access for the insertion of a medical device. Drug relief ideal delivery throughout the pocket with prolonged duration; that are provided by gingival crevicular fluid (GCF), which plays as the leaching medium $^{(4)}$

Semisolid gels have many advantages as local delivery systems. They are relatively simple to prepare and manage though they have a rapid drug release rate. As well as, they are very biocompatible and bio-adhesive, thus simply adhere to the mucosa in the periodontal pocket ${ }^{(5)}$. Metformin (MF) as an antidiabetic drug can excess the peripheral load of glucose and reducing the production of hepatic glu$\operatorname{cose}^{(6)}$. Further, it may modify the inflammatory state independently of its role on glycemic state ${ }^{(7)}$. The anti-inflammatory process of MF has been assured on several cells; such as human vascular smooth muscle cells and endothelial cells ${ }^{(8)}$. Currently, MF has anti-inflammatory effect to chronic periodontitis and the implied mechanisms remains to be totally demonstrated ${ }^{(9)}$. Statins are the generally prescribed drugs to hold the serum cholesterol levels to decrease the risk of cardiovascular ailments. Among various statins, simvastatin (SV) has importance in the field of periodontal treatment ${ }^{(10)}$.
Simvastatin is a synthetic statin holding several effects including reducing the bone resorption ${ }^{(11)}$. Moreover, it has anti-inflammatory actions as they are able to repress expression and reactions of superficial molecules on leukocytes. Simvastatin prevents migration of neutrophils, reduce expression of inter-cellular adhesion molecule-1 in monocytes and macrophages ${ }^{(12)}$.

The Interlukin-6 (IL-6) works to control the spread of tissue inflammatory responses. In prolonged illness, commonly exemplified by immune stressors such as chronic intracellular infections and tumors. IL-6 acts as a promotor of intense phase reactions and also, it is a player in extract the cellular immune responses to affected cells and mucosal humoral responses conducted against repeated infection $^{(13)}$. The Tumor Necrosis Factor-alpha (TNF- $\alpha$ ) is act as a proinflammatory cytokine that is often overexpressed in serious illness such as sepsis syndrome, rheumatoid arthritis, inflammatory bowel disease and periodontitis ${ }^{(14)}$. TNF- $\alpha$ acts an important role in periodontal illness in prolonged periodontitis cases. Its expression was elevated in the GCF at sites where bone and attachment loss have just occurred. Likewise, TNF- $\alpha$ has a pivotal function in bone pathophysiology because it activates the bone resorption and reduces the bone formation as it acts as stimulator for the osteoclasts which mediate bone resorption. Also, it known to prevent osteoblast function and differentiation ${ }^{(15)}$.

Till date, finite research has been carried out to appraise the effects of local adjunct of SV or MF on the biochemical mediators of inflammation ${ }^{(16-19)}$. Hence the study was endeavored to evaluate the adequacy of $1.2 \% \mathrm{SV}$ and $1 \%$ MF gel which play as a local delivery medication in adjunct to scaling and root planning (SRP) on GCF IL-6 and TNF- $\alpha$ levels in chronic periodontitis patients and correlating their values with clinical parameters. 


\section{PATIENTS AND METHODS}

The current trial was a randomized, single-center, longitudinal and triple-masked (patients, investigators and statistician) study. It was managed overall the 9 months; in which recordings were set at baseline, 3, 6 and 9 months. The study protocol was authorized by the Ethical Committee, Faculties of Dentistry, Al-Azhar University. All eligible patients were thoroughly learned of the nature, potential risks and aids of their participation in the research and signed their informed approval documents.

\section{Patient selection}

This study was designed as randomized clinical study, included 45 systemically healthy chronic periodontitis patients (20 males and 25 females, ranged in age from 18 to 38 years with mean age of $23 \pm 2.8$ years) with mild to moderate chronic periodontitis build on the approved grades of the periodontal diseases. All patients were selected from those visiting the outpatient clinic, Oral Medicine and Periodontology Department, Faculty of Dentistry, Al-Azhar University, Assiut Branch.

\section{Inclusion criteria}

The patients were volunteers with sites showing 3-5 mm pocket depth, 1-3 mm clinical attachment loss (CAL), with horizontal or vertical bone loss on intraoral periapical radiographs and no past of periodontal therapy or use of antibiotics in the last 6 months.

\section{Exclusion criteria}

All the following were excluded; patients with known systemic disease, with known or suspected allergy to any drugs, who used of tobacco in any form, alcoholic, immunocompromised patients, pregnant or lactating females, patients with periodontal diseases rather than chronic periodontitis and patients received antibiotics or anti-inflammatory agents after therapy for 9 months.

\section{Randomization and blindness:}

The random number table was generated by specific software. Numbers were concealed in closed envelopes and each patient select own number which referred to specific group. Neither the patient nor the controller was aware of the nature of additives tested.

\section{Sample size calculation and power analysis}

For the sample size calculation, the power analysis was performed using $\mathrm{G}$ Power system (Ver. 3.192 copy right 1992-2020) for a one-way locked effect analysis of variance (ANOVA). The criterion for significance was set at $\alpha=0.05$ (type I error) and $\beta=0.20$ (type II error) to recognize significant difference (Q) of $1 \mathrm{~mm}$ between groups considering the change in CAL as the primary outcome variable, with a 95\% confidence interval. Therefore, the required sample size was found to be 15 patients in each group with $0.97 \%$ actual power of this study was achieved.

\section{Patient grouping}

Forty-five patients were randomly divided into the placebo, SV and MF groups. Fifteen patients were assigned to each group. Careful instructions on proper oral hygiene measures were given to each patient. For each patient SRP was done at baseline. Group I patients were treated with SRP pursued by placebo gel. Group II with SRP treatment and application $1.2 \% \mathrm{SV}$ gel. Group III were treated with SRP and application $1 \%$ MF gel.

\section{Preparation of simvastatin, metformin and placebo}

The simvastatin, metformin and placebo gel were prepared in the Faculty of Pharmacology, AlAzhar University, Assiut, Branch. Gel was prepared by adding $2.5 \mathrm{~g}$ of methylcellulose to $100 \mathrm{~g}$ of grade water slowly and stirring continuously to attain the gel consistency. Once this was prepared; $1.2 \mathrm{~g}$ of $\mathrm{SV}$ or $1 \mathrm{~g}$ MF was combined gradually with regular stirring to get the preparation. The placebo gel 
was processed by the same manner excepting that effective materials was not added. The prepared gel was give it to the laboratory analysis to establish the proportion of SV at $1.2 \%$ and MF at $1 \%$.

\section{Local drug delivery}

Gel was implanted within the periodontal pockets using a syringe with a rounded cannula (Figure 1). Patients were advised to stop from chewing hard or sticky foods, brushing near the treated field, or applying any interdental care for a week. Adverse effects were notable at the next regular appointments and any supra-gingival deposits were detached.

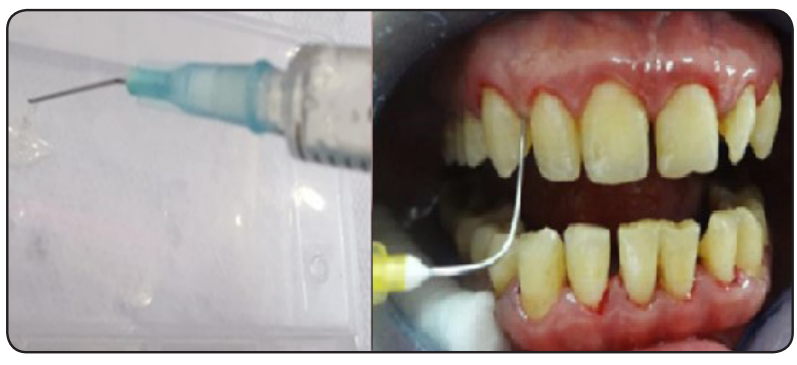

Fig. (1) Showing Gel Formulation (at Left Side) and IntraPocket Application (at Right Side).

\section{Periodontal evaluation}

The periodontal conditions of each patient were evaluated at baseline, 3, 6 and 9 months after treatment. Plaque Index (PI) used to assess plaque accumulation around gingival margin. Gingival Index (GI) used to assess gingival inflammation ${ }^{(20,21)}$. Probing Depth (PD) and Clinical Attachment Level (CAL) were listed by William's graduated periodontal probe ${ }^{(22,23)}$.

\section{Gingival crevicular transudate sample collection and preparation}

Gingival crevicular fluid sample was taken prior to periodontal therapy as well as 3, 6 and 9 months' follow-up. The deepest three sites with PD $\leq 5 \mathrm{~mm}$ and $\mathrm{CAL} \leq 4 \mathrm{~mm}$ were selected for $\mathrm{GCF}$ sampling(24). Supra-gingival plaque was ejected and the site was then dried smoothly with an air syringe. Standardized paper point size \#30 was inserted into the crevice until mild resistance was felt. The paper point was left in pocket for 30 seconds $^{(25)}$. Paper points which were tarnished with blood and saliva were rejected. The collected GCF samples were pooled in an Eppendorf tube and diluted in phosphate buffer saline up to $650 \mu \mathrm{l}$ and transported to the laboratory. Following $20 \mathrm{~min}$, shaking paper points were evacuated and centrifuged for $5 \mathrm{~min}$. at $5800 \mathrm{~g}$ to remove plaque and cellular elements. The specimens were frozen at $-20^{\circ} \mathrm{C}$ till they were examined for IL- 6 and TNF- $\alpha$.

\section{Quantification of IL-6 and TNF- $\alpha$}

Samples were checked by enzyme-linked immunosorbent assay (ELISA) to resolve the levels of IL-6 according to the manufacturer's instructions. It occupied a quantitative sandwich enzyme immunoassay manner. ELISA reader was used to record the optical density of the tested samples. The quantity of interleukin sample was calculated by comparing with the standard calibrated curve included in the assay kit. The concentration of interleukin in each sample was determined by split the amount of interleukin by the volume of the sample $(\mathrm{pg} / 100 \mu \mathrm{l})$.

\section{Statistical analysis}

Data were recorded, computed, tabulated and analyzed at a significance level of $\mathrm{P}<0.05$ by GraphPad Prism (version 8, San Diego, California). The mean and standard deviation amount were estimated for each group in each test. For parametric statistics; repeated measure ANOVA was used to compare among more than two groups in linked samples. Graphs were achieved by the Microsoft excel data.

\section{RESULTS}

\section{Oral hygiene status}

This clinical trial was operated in 45 patients get mild to moderate chronic periodontitis; whom equally treated by nonsurgical periodontal therapy in association with placebo, SV or MF. There was significant advancement of plaque amount 
and gingival tissue condition in all 3 groups from onset of study until 9 months. At baseline (T0) observation between all 3 groups showed the mean variations in the clinical parameters scores (PI and GI) were statistically not significant due to the common selection criteria. At 6 and 9 months (T2 and T3), PI and GI showed statistically significant reduction in group II and III than group I. Among all the 3 groups; MF group showed higher decrease in GI score as compared to placebo, SV groups at 6 and 9 months (Table 1, Chart 1).

\section{Pocket depth and clinical attachment loss reduction}

The reduction of PD and CAL were statistically significant within all 3 groups from beginning of study until 9 months. When comparing SRP vs. SV intended, the reduction in PD and CAL at each investigation period was not statistically significant. The PD reduction was statistically significant, when comparing SRP vs. MF and SV vs. MF at 6 and 9 months (Table 2, Chart 2). The CAL decrease was statistically significant when comparing SRP vs. MF and SV vs. MF at 3, 6 and 9 months. Forasmuch, MF group gave better effects at reduction of PD and CAL.

TABLE (1) Mean \pm SD of Plaque Index and Gingival Index, Intragroup ANOVA Test for Comparison Plaque Index and Gingival Index between Treatment Intervals within each Group and Intergroup ANOVA Test for Comparison between Studied Groups within each Interval. T0 = Baseline, T1= 3 Months T2 $=6$ Months, T3 = 9 Months. $(\mathrm{p} \leq 0.05=$ Significant $)$

\begin{tabular}{|c|c|c|c|c|c|c|c|c|c|c|c|c|c|c|c|c|}
\hline \multirow{2}{*}{ Visits } & \multicolumn{8}{|c|}{ Plaque Index } & \multicolumn{8}{|c|}{ Gingival Index } \\
\hline & \multicolumn{4}{|c|}{ Group 1} & \multicolumn{2}{|c|}{ Group 2} & \multicolumn{2}{|c|}{ Group 3} & \multicolumn{4}{|c|}{ Group 1} & \multicolumn{4}{|c|}{ Group 3} \\
\hline T0 & \multicolumn{4}{|c|}{$2.4 \pm 0.32$} & \multicolumn{2}{|c|}{$2.6 \pm 0.41$} & \multicolumn{2}{|c|}{$2.6 \pm 0.53$} & \multicolumn{4}{|c|}{$2.6 \pm 0.43$} & \multicolumn{4}{|c|}{$2.7 \pm 0.50$} \\
\hline T1 & \multicolumn{4}{|c|}{$1.4 \pm 0.13$} & \multicolumn{2}{|c|}{$1.4 \pm 0.30$} & \multicolumn{2}{|c|}{$1.2 \pm 0.20$} & \multicolumn{4}{|c|}{$1.3 \pm 0.065$} & \multicolumn{4}{|c|}{$0.99 \pm 0.10$} \\
\hline T2 & \multicolumn{4}{|c|}{$1.2 \pm 0.14$} & \multicolumn{2}{|c|}{$1.0 \pm 0.23$} & \multicolumn{2}{|c|}{$0.87 \pm 0.19$} & \multicolumn{4}{|c|}{$1.0 \pm 0.12$} & \multicolumn{4}{|c|}{$0.42 \pm 0.09$} \\
\hline T3 & \multicolumn{4}{|c|}{$0.78 \pm 0.13$} & \multicolumn{2}{|c|}{$0.79 \pm 0.25$} & \multicolumn{2}{|c|}{$0.57 \pm 0.29$} & \multicolumn{4}{|c|}{$0.53 \pm 0.067$} & \multicolumn{4}{|c|}{$0.30 \pm 0.04$} \\
\hline & \multicolumn{16}{|c|}{ Intragroup ANOVA Test } \\
\hline Groups & \multicolumn{4}{|c|}{$\mathrm{F}$} & \multicolumn{4}{|c|}{$\mathrm{p}$} & \multicolumn{5}{|c|}{$\mathrm{F}$} & & $\mathrm{p}$ & \\
\hline Group I & & & & & & 0.0 & & & & & 226. & & & & $0.00^{* *}$ & \\
\hline Group II & & & & & & 0.0 & & & & & 236. & & & & $0.00^{* *}$ & \\
\hline Group III & & & & & & 0.0 & & & & & 286.2 & & & & $0.00^{* *}$ & \\
\hline & $\mathrm{TO}$ & & & 1 & & $\Gamma 2$ & & $\Gamma 3$ & $\mathrm{~T}$ & & & $\Gamma 1$ & & $\lceil 2$ & & Т3 \\
\hline ANOVA Test & F & $\mathrm{p}$ & F & $\mathrm{p}$ & $\mathrm{F}$ & $\mathrm{p}$ & $\mathrm{F}$ & $\mathrm{p}$ & $\mathrm{F}$ & $\mathrm{p}$ & $\mathrm{F}$ & $\mathrm{p}$ & F & $\mathrm{p}$ & $\mathrm{F}$ & $\mathrm{p}$ \\
\hline & 0.4 & 0.6 & 2 & 0.1 & 30.4 & $0.00^{* *}$ & 4.2 & $0.02^{* *}$ & 1.8 & 0.6 & 23 & $0.00^{* *}$ & 54.8 & $0.00^{* *}$ & 30 & $0.00^{* *}$ \\
\hline SRP vs. SV & 0.7 & & & & & $00^{* *}$ & & 9.9 & 0. & & & .20 & & 43 & & $02 * *$ \\
\hline SRP vs. MF & 0.6 & & & 1 & & $00^{* *}$ & & $4^{* *}$ & 0. & & & $0^{* *}$ & & $0^{* *}$ & & $00 * *$ \\
\hline SV vs. MF & 0.9 & & & & & .1 & & $3^{* *}$ & 0.5 & & & 0 0** & & $0^{* *}$ & & $00^{* *}$ \\
\hline
\end{tabular}


TABLE (2) Mean \pm SD of Pocket Depth and Clinical Attachment Loss, Intragroup ANOVA Test for Comparison Pocket Depth and Clinical Attachment Loss between Treatment Intervals within each Group and Intergroup ANOVA Test for Comparison between Studied Groups within each Interval. $(\mathrm{p} \leq 00.05) \mathrm{T} 0=$ Baseline, $\mathrm{T} 1=3$ Months, $\mathrm{T} 2=6$ Months $\mathrm{T} 2=9$ Months.

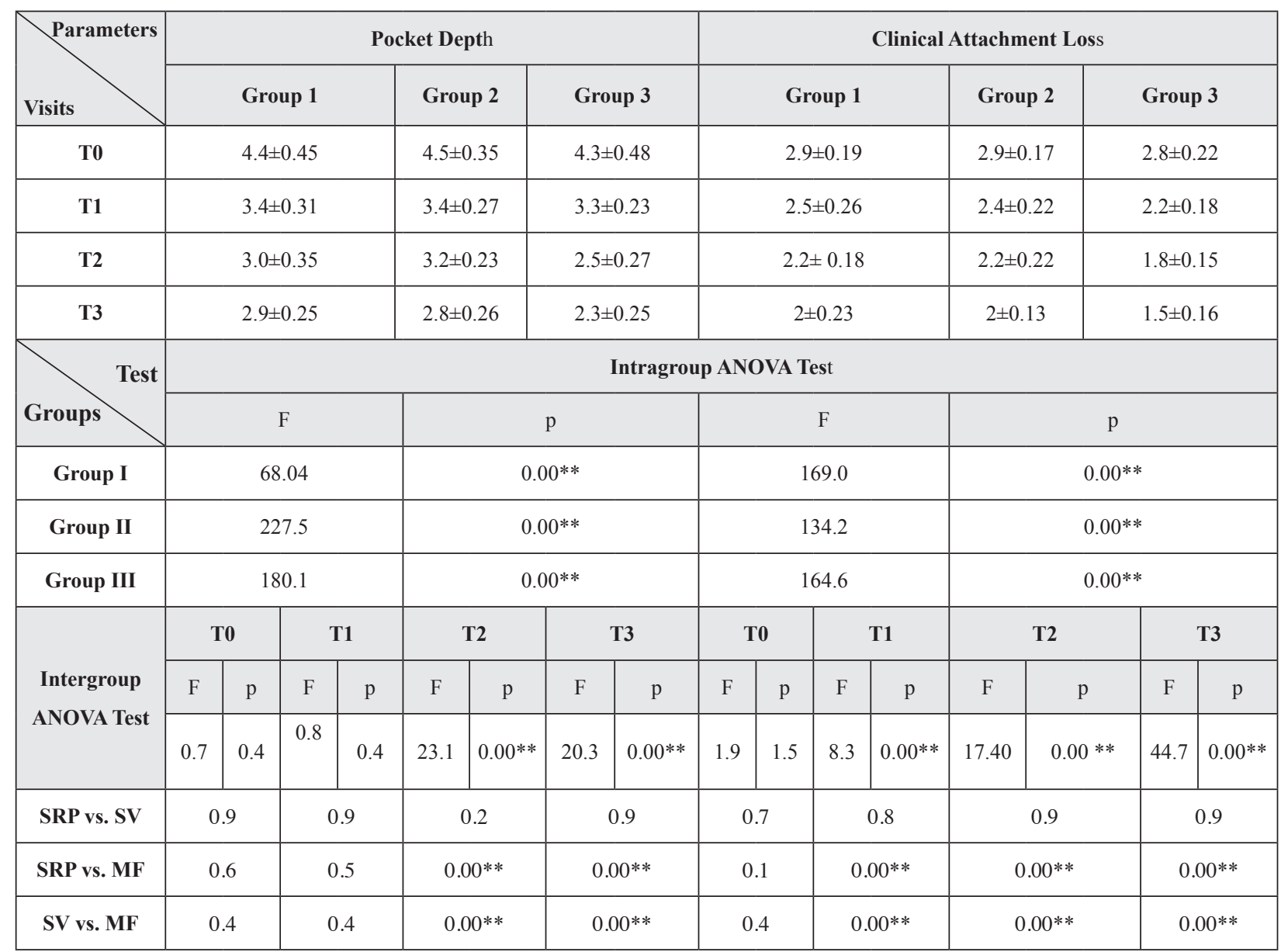

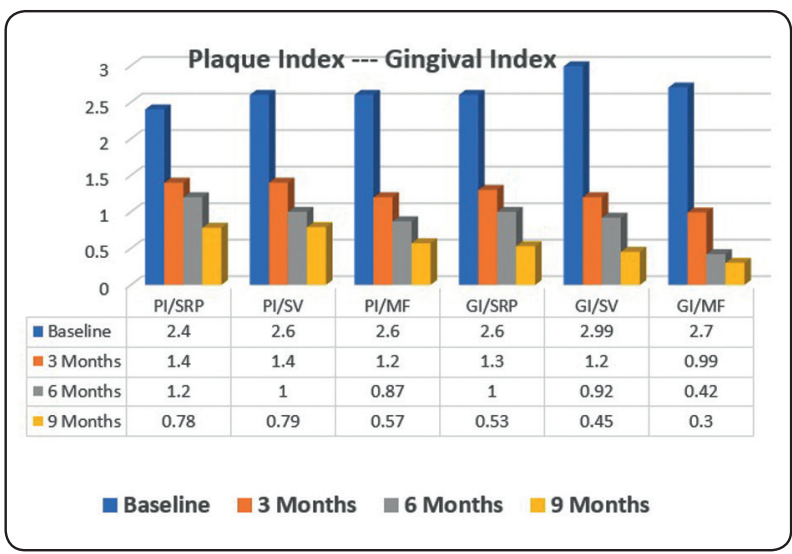

Chart (1) Means of PI and GI of Treatment Groups at Interval Periods.

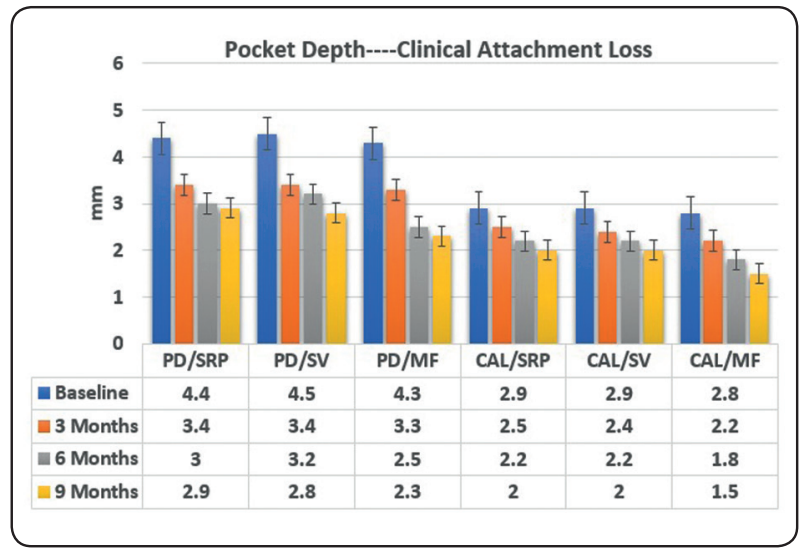

Chart (2) Means of PD and CAL of Treatment Groups at Interval Periods. 
TABLE (3) Mean \pm SD of IL-6 and TNF- $\alpha$, Intragroup ANOVA Test for Comparison IL-6 and TNF- $\alpha$ between Treatment Intervals within each Group and Intergroup ANOVA Test for Comparison between Studied Groups within each Interval. $(\mathrm{p} \leq 00.05) \mathrm{T} 0=$ Baseline, $\mathrm{T} 1=3$ Months, T2 $=6$ Months T2 $=9$ Months. Finally, Correlation between Changes of IL- 6 \& TNF- $\alpha$ Levels from Baseline to 9 Months in All Groups Was Reported.

\begin{tabular}{|c|c|c|c|c|c|c|c|c|c|c|c|c|c|c|c|c|}
\hline \multirow{2}{*}{ Visits } & \multicolumn{8}{|c|}{ IL-6 } & \multicolumn{8}{|c|}{ TNF- $\alpha$} \\
\hline & \multicolumn{3}{|c|}{ Group 1} & \multicolumn{3}{|c|}{ Group 2} & \multicolumn{2}{|c|}{ Group 3} & \multicolumn{3}{|c|}{ Group 1} & \multicolumn{3}{|c|}{ Group 2} & \multicolumn{2}{|c|}{ Group 3} \\
\hline T0 & \multicolumn{3}{|c|}{$1340 \pm 52.14$} & \multicolumn{3}{|c|}{$1383 \pm 83.13$} & \multicolumn{2}{|c|}{$1310 \pm 113.8$} & \multicolumn{3}{|c|}{$341.3 \pm 34.23$} & \multicolumn{3}{|c|}{$351.7 \pm 37.83$} & \multicolumn{2}{|c|}{$369 \pm 38.16$} \\
\hline T1 & \multicolumn{3}{|c|}{$969.1 \pm 49.22$} & \multicolumn{3}{|c|}{$857.4 \pm 43.43$} & \multicolumn{2}{|c|}{$743.3 \pm 49.41$} & \multicolumn{3}{|c|}{$299.2 \pm 30.57$} & \multicolumn{3}{|c|}{$251.4 \pm 32.19$} & \multicolumn{2}{|c|}{$236.8 \pm 22.48$} \\
\hline $\mathbf{T} 2$ & \multicolumn{3}{|c|}{$873.8 \pm 22.54$} & \multicolumn{3}{|c|}{$781.9 \pm 33.60$} & \multicolumn{2}{|c|}{$683.7 \pm 36.03$} & \multicolumn{3}{|c|}{$283.5 \pm 28.73$} & \multicolumn{3}{|c|}{$230.9 \pm 27.56$} & \multicolumn{2}{|c|}{$216.4 \pm 15.64$} \\
\hline $\mathbf{T 3}$ & \multicolumn{3}{|c|}{$855.8 \pm 24$} & \multicolumn{3}{|c|}{$757.7 \pm 40.69$} & \multicolumn{2}{|c|}{$650 \pm 41.76$} & 273 & $6 \pm 27$ & & $216.2 \pm$ & 22.75 & & 178.6 & $6 \pm 23.26$ \\
\hline Test & & & & & & & & ragroup & NOY & A Tes & & & & & & \\
\hline Groups & & & F & & & 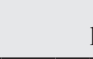 & & & & & & & & $\mathrm{p}$ & & \\
\hline Group I & & & 52.3 & & & 0.0 & $* *$ & & & & & & & $0.00 *$ & & \\
\hline Group II & & & 82.4 & & & 0.0 & *** & & & & & & & $0.00^{*}$ & & \\
\hline Group III & & & 47.7 & & & 0.0 & $* *$ & & & & & & & $0.00^{*}$ & & \\
\hline & & 0 & & $\Gamma 1$ & & 2 & & 3 & T & & $\mathbf{T}$ & & $\mathbf{T}$ & 2 & & T3 \\
\hline Intergroup & F & $\mathrm{p}$ & $\mathrm{F}$ & $\mathrm{p}$ & $\mathrm{F}$ & $\mathrm{p}$ & $\mathrm{F}$ & $\mathrm{p}$ & F & $\mathrm{p}$ & $\mathrm{F}$ & $\mathrm{p}$ & $\mathrm{F}$ & $\mathrm{p}$ & $\mathrm{F}$ & $\mathrm{p}$ \\
\hline & 2.5 & 0.09 & 89.7 & $0.00 * *$ & 192.4 & $0.00 * *$ & 159.1 & $0.00 * *$ & 2.3 & 0.1 & 20.1 & $0.00 * *$ & 32.5 & $0.00 * *$ & 59.4 & $0.00 * *$ \\
\hline SRP vs. SV & 0 & .3 & & $0 * *$ & & $0 * *$ & & $0 * *$ & 0 & & & & & $.00 * *$ & & $00 * *$ \\
\hline SRP vs. MF & 0 & .7 & & $0 * *$ & & $0 * *$ & & $0 * *$ & 0. & & & & & $.00 * *$ & & $00 * *$ \\
\hline SV vs. MF & 0. & 07 & & $0 * *$ & & $0 * *$ & & $0 * *$ & 0 & & & & & 0.2 & & $00 * *$ \\
\hline & & Correl & ation 1 & etween $\mathrm{C}$ & hanges & of IL-6 & TNF-C & Levels & om I & aselin & to $9 \mathrm{M}$ & nths in $A$ & Il Gro & ups. & & \\
\hline & & & & oup I & & & & Gro & Ip II & & & & & Group II & & \\
\hline IL-6 & & $r-v$ & alue & & P-value & & r- value & & & -value & & & alue & & P-val & \\
\hline & & 0.9 & 88 & & $0.01 * *$ & & 0.994 & & & $005^{* *}$ & & & 983 & & $0.01^{\prime}$ & \\
\hline
\end{tabular}

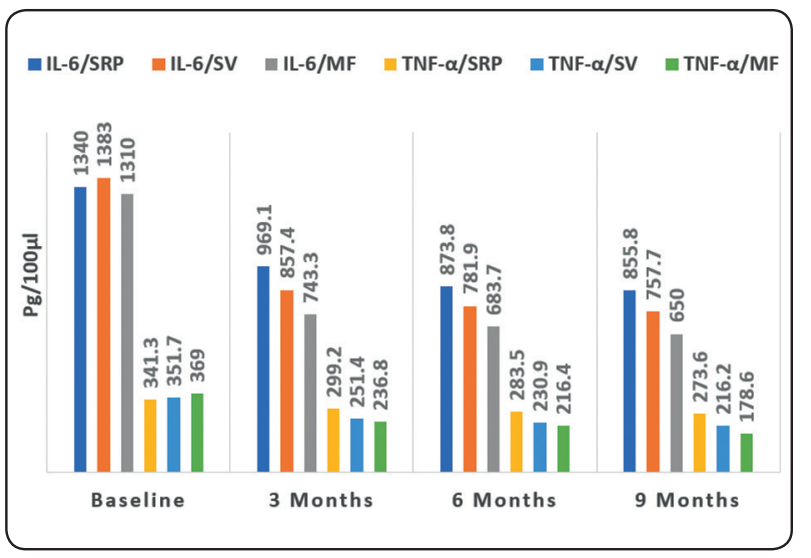

Chart (3) Means of IL-6 and TNF- $\alpha$ of Treatment Groups at Interval Periods.

\section{IL-6 and TNF- $\alpha$ levels}

Significant reduction in IL-6 and TNF- $\alpha$ levels appeared from baseline to the end of testing period in all groups. Inter-groups ANOVA test showed statistically significant difference between groups at 3, 6 and 9 months (Table 3, Chart 3). High significant contraction in IL- 6 and TNF- $\alpha$ levels recorded in MF group.

\section{Correlations}

Statistically significant clear correlation was established between IL- 6 and TNF- $\alpha$ levels. 


\section{DISCUSSION}

Scaling and root planning efficacy has limitations and complete healing may not be achieved at sites with a PD $>4 \mathrm{~mm}$. additional periodontal therapies may be necessary if deep probing depths persist ${ }^{(3)}$. Local Delivery drugs have numerous advantages over systemic agents including minimally invasive, direct application at the site of infection, avoidance of gastro-intestinal issues, devaluation in the dose, frequency of drug administration and have potential role in improving the clinical parameters associated with periodontitis ${ }^{(26,27)}$. Therefore, in the current study, it had 1.2\% SV and 1\% MF in situ gel formulation to assess and compare clinically and biochemically with a controlled drug release.

Anti-inflammatory, antioxidant, antimicrobial and osteostimulative properties of statins and MF were inferred by decline in the expression of TNF- $\alpha$ plasma concentrations and inhibit the signaling pathway for IL-1- and IL-6- interfere inflammation ${ }^{(28,29)}$. Also, MF exerts anti-inflammatory action via restoring the endothelial function, increased nitric oxide synthesis through stimulation of AMP activated protein kinase and decreased reactive oxygen species production through inhibition of nicotinamide adenine dinucleotide phosphate oxidase ${ }^{(30)}$.

Results of this clinical study viewed an improvement in plaque control and gingival tissue health in all groups from the start to the end of observation period; this reduction of PI and GI was statistically significant when correlate MF group against $\mathrm{SV}$ group at 6 and 9 months. These results in correspondence with Kurian et al. ${ }^{(31)}$ study showed significant reduction in PI and GI at all intervals. Another study performed by Pankaj et al. ${ }^{(32)}$ to compare the performance of subgingival delivered $1.2 \% \mathrm{SV}$ and $1 \% \mathrm{MF}$ gel showed that greater reduction GI like present results of this study.

The measurements of PD and CAL in the current trial showed statistically significant reduction in all groups; this liked study of Pradeep et al. ${ }^{(33)}$ they mentioned that bone filling in intrabony defects, thereby decreasing PD and increasing attachment gain in chronic patients. In contrary to results of this study which showed significant reduction of PD and CAL in MF group greater than SV group; study of Pankaj et al. ${ }^{(32)}$ showed that within the limitations of the trial; there was a significant improvement in clinical parameters such as PD reduction and CAL gain in the $1.2 \% \mathrm{SV}$ group as compared to the $1 \% \mathrm{MF}$ and placebo groups. Furthermore, Chen et al. ${ }^{(34)}$ stated that statins more effectively reduce inflammation when it compared with MF. Quite the opposite of their conclusions; present trial showed more reduction of inflammatory biomarkers (IL-6 and TNF- $\alpha$ ) in MF group greater than SV and placebo groups. The results of current study were consistent with Tan et al. ${ }^{(35)}$ they concluded that MF had protective effects on porphyromonas gingivalis promoted inflammation and inhibited the activation of NLRP3 inflammasome and the secretion of IL$1 \beta$ and IL-18. Additionally, NF- $x$ B pathway related genes and TNF- $\alpha$ related genes were found to be involved in the anti-inflammatory effects of MF. Metformin conducting NLRP3 inflammasome could possibly be service for the prevention and treatment of porphyromonas gingival is related periodontal diseases.

In current application, significant clear correlation between IL- 6 and TNF- $\alpha$ was recorded; these findings were in contrary with Noh et al..$^{(36)}$ they mentioned that the TNF- $\alpha$ level was not correlated with the IL-6 in chronic periodontitis patients. Forthcoming studies should revolve around effects of statins and MF in pro/anti-inflammatory mediators in GCF and periodontal tissues in clinical trials. Also, their effects in bone regeneration in histobiochemical and advanced radiographic analysis.

\section{CONCLUSION}

Within the limitations, this study showed both $1.2 \% \mathrm{SV}$ and $1 \% \mathrm{MF}$ are used as an effective local adjunct therapy. However, MF comparatively better than SV in respect of clinical and biochemical markers. 


\section{RECOMMENDATIONS}

1. The need for more clinical and biochemical studies in extensive forms of periodontitis for more extended period to measure the exact effects of simvastatin and metformin on periodontal tissues, especially measuring osteogenic and anti- inflammatory mediators.

2. Other than local application of simvastatin and metformin, studies, to surgical applications in intrabony defects, socket preservation and treatment of peri- implantitis, were recommended to evaluated maximum benefits of these promising materials.

\section{REFERENCES}

1. Curtis MA, Diaz PI, Van Dyke TE. The role of the microbiota in periodontal disease. Periodontol 2000. 2020;83(1):14-25.

2. Aimetti M. Nonsurgical periodontal treatment. Int J Esthet Dent. 2014;9(2):251-67.

3. Geisinger ML, Kaur M, Basma H. Nonsurgical periodontal therapy: a review of current standards of care and innovations to improve gingival and periodontal health. Current Oral Health Reports. 2019;6(3):177-87.

4. Garg T, Singh O, Arora S, Murthy R. Scaffold: a novel carrier for cell and drug delivery. Crit Rev Ther Drug Carrier Syst. 2012;29(1):1-63.

5. Garg T, Rath G, Goyal AK. Comprehensive review on additives of topical dosage forms for drug delivery. Drug delivery. 2015;22(8):969-87.

6. Sansome DJ, Xie C, Veedfald S, Horowitz M, Rayner CK, $\mathrm{Wu}$ T. Mechanism of glucose-lowering by metformin in type 2 diabetes: Role of bile acids. Diabetes Obes Metab. 2020;22(2):141-8.

7. Zaikina T, Babadjan V, Ryndina N, Borzova O, Kovalyova Y. Influence of the metformin therapy on the activity of endothelial-dependent mediators among patients with acute myocardial infarction and concomitant type 2 diabetes mellitus. Georgian Med News. 2018;(274):92-7.

8. Ni HZ, Liu Z, Sun LL, Zhou M, Liu C, Li WD, et al. Metformin inhibits angiogenesis of endothelial progenitor cells via miR-221-mediated p27 expression and autophagy. Future Med Chem. 2019;11(17):2263-72.
9. Kang W, Wang T, Hu Z, Liu F, Sun Y, Ge S. Metformin inhibits Porphyromonas gingivalis lipopolysaccharideinfluenced inflammatory response in human gingival fibroblasts via regulating activating transcription factor-3 expression. J periodontol. 2017;88(10):e169-78.

10. du Souich P, Roederer G, Dufour R. Myotoxicity of statins: mechanism of action. Pharmacol Ther. 2017;175:1-16.

11. Kinra P, Khan S. Simvastatin: its potential new role in periodontal regeneration. Biol Med. 2011;3:215-21.

12. Feng Y, Lei B, Zhang F, Niu L, Zhang H, Zhang M. Antiinflammatory effects of simvastatin during the resolution phase of experimentally formed venous thrombi. J Investig Med. 2017;65(6):999-1007.

13. Zhang H, Sun B. Pleiotropic regulations of neutrophil receptors response to sepsis. Inflamm Res. 2017;66(3):197207.

14. Holdsworth SR, Gan PY. Cytokines: names and numbers you should care about. Clin J Am Soc Nephrol. 2015;10(12):2243-54.

15. Jiang ZL, Cui YQ, Gao R, Li Y, Fu ZC, Zhang B, et al. Study of TNF- $\alpha$, IL-1 $\beta$ and LPS levels in the gingival crevicular fluid of a rat model of diabetes mellitus and periodontitis. Dis Markers. 2013;34(5):295-304.

16. Grover HS, Kapoor S, Singh A. Effect of topical simvastatin $(1.2 \mathrm{mg})$ on gingival crevicular fluid interleukin-6, interleukin-8 and interleukin-10 levels in chronic periodontitis-a clinic biochemical study. J Oral Biol Craniofac Res. 2016;6(2):85-92.

17. Rath A, Mahenra J, Thomas L, Sandhu M, Namasi A, Ramakrishna T. A clinical, radiological and IL-6 evaluation of subgingivally delivered simvastatin in the treatment of chronic periodontitis. Int J Drug Deliv. 2012;4(1):70-8.

18. de Araujo AA, Pereira AD, de Medeiros CA, de Castro Brito GA, de Carvalho Leitao RF, de Souza Araujo L, et al. Effects of metformin on inflammation, oxidative stress, and bone loss in a rat model of periodontitis. PloS one. 2017;12(8):e0183506.

19. Vemanaradhya GG, Emani S, Mehta DS, Bhandari S. Effect of $1.2 \%$ of simvastatin gel as a local drug delivery system on gingival crevicular fluid interleukin- 6 \& interleukin-8 levels in non-surgical treatment of chronic periodontitis patients. Arch Oral Biol. 2017;82:55-61.

20. Niemiec BA. Periodontal disease. Top Companion Anim Med. 2008;23(2):72-80.

21. Loe H. The gingival index, the plaque index and the retention index systems. J Periodontol.1967;38:610-16. 
22. Polson A, Caton J, Yeaple R, Zander H. Histologic determination of probe tip penetration into gingival sulcus of humans using an electronic pressure sensitive probe. J Clin Periodontol. 1980;7:479-88.

23. Donos N. The periodontal pocket. Periodontol 2000. 2018;76(1):7-15.

24. Moriya Y, Obama T, Aiuchi T, Sugiyama T, Endo Y, Koide $\mathrm{Y}$, et al. Quantitative proteomic analysis of gingival crevicular fluids from deciduous and permanent teeth. J Clin Periodontol. 2017;44(4):353-62.

25. Taylor JJ, Preshaw PM. Gingival crevicular fluid and saliva. Periodontol 2000. 2016;70(1):7-10.

26. Da Rocha HA, Silva CF, Santiago FL, Martins LG, Dias PC, De Magalhaes D. Local drug delivery systems in the treatment of periodontitis: a literature review. J Int Acad Periodontol. 2015;17(3):82-90.

27. Rajeshwari HR, Dhamecha D, Jagwani S, Rao M, Jadhav $\mathrm{K}$, Shaikh $\mathrm{S}$, et al. Local drug delivery systems in the management of periodontitis: A scientific review. J Control Release. 2019;307:393-409.

28. Alam S, Ueki K, Nakagawa K, Marukawa K, Hashiba Y, Yamamoto E, et al. Statin-induced bone morphogenetic protein (BMP) 2 expression during bone regeneration: an immunohistochemical study. Oral Surg Oral Med Oral Pathol Oral Radiol Endod. 2009;107(1):22-29.

29. Omoigui S. The Interleukin-6 inflammation pathway from cholesterol to aging-role of statins, bisphosphonates and plant polyphenols in aging and age-related diseases. Immun Ageing. 2007;4:1.
30. Saisho Y. Metformin and inflammation: its potential beyond glucose-lowering effect. Endocr Metab Immune Disord Drug Targets. 2015;15:196-205.

31. Kurian IG, Dileep P, Ipshita S, Pradeep AR. Comparative evaluation of subgingivally-delivered $1 \%$ metformin and aloe vera gel in the treatment of intrabony defects in chronic periodontitis patients: a randomized, controlled clinical trial. J Investig Clin Dent. 2018;9(3):e12324.

32. Pankaj D, Sahu I, Kurian IG, Pradeep AR. Comparative evaluation of subgingivally delivered $1.2 \%$ rosuvastatin and $1 \%$ metformin gel in treatment of intrabony defects in chronic periodontitis: A randomized controlled clinical trial. J Periodontol. 2018;89(11):1318-25.

33. Pradeep AR, Karvekar S, Nagpal K, Patnaik K, Guruprasad CN, Kumaraswamy KM. Efficacy of locally delivered $1.2 \%$ rosuvastatin gel to non-surgical treatment of patients with chronic periodontitis: a randomized, placebocontrolled clinical trial. J Periodontol. 2015;86(6):738-45.

34. Chen HH, Lin MC, Muo CH, Yeh SY, Sung FC, Kao CH. Combination therapy of metformin and statin may decrease hepatocellular carcinoma among diabetic patients in Asia. Medicine. 2015;94(24):e1013.

35. Tan Y, Chen J, Jiang Y, Chen X, Li J, Chen B, et al. The antiperiodontitis action of metformin via targeting NLRP3 inflammasome. Archives of Oral Biology. 2020:114;104692.

36. Noh MK, Jung M, Kim SH, Lee SR, Park KH, Kim DH, et al. Assessment of IL-6, IL-8 and TNF- $\alpha$ levels in the gingival tissue of patients with periodontitis. Exp Ther Med. 2013;6(3):847-51. 